No. $14(18)$

\title{
TILING BY SQUARES, KIRCHHOFF'S LAWS AND A FLOW PROBLEM IN THE ECONOMY
}

\begin{abstract}
Arkadiusz Maciuk, Antoni Smoluk
Abstract. The problem of tiling by squares was formulated more than one hundred years ago and has been mostly regarded as an interesting mathematical issue in geometry, yet without any special practical associations. In addition to a historical outline of the problem, this paper demonstrates how Smith's 1940 method using digraphs helps associate an issue linked to two-dimensional dissection or packaging with such economic problems as transportation network planning, researching linkages between suppliers and the like. We also discuss simple perfect squared rectangles whose relevant laws of nature are as yet unknown, according to the principle that elegant scientific structures sooner or later find their equivalents in the real world.
\end{abstract}

Keywords: squarings, digraph, Kirchhoff's laws, flow problem in the economy.

JEL Classification: B16, C02

DOI: $10.15611 / \mathrm{dm} .2017 .14 .04$

\section{Introduction}

The history of dissecting a rectangle into squares dates back to the beginnings of the 20th century. Max Dehn, a German mathematician, famous among others for resolving in 1900 Hilbert's third problem on the congruence of polyhedra, published a paper dealing with the dissection of rectangles into squares [Dehn 1903]. Assuming certain properties of some systems of linear equations, he proved that a rectangle can be squared if and only if its sides are commensurable, and if a rectangle can be squared, then there are infinitely many perfect squarings. In 1924, the problem of dissecting a rectangle into different squares was introduced by Stanisław Ruziewicz, a professor at the University of Lviv, whose assistant, Zbigniew Moroń, resolved this problem in 1925 [Moroń 1925]. Roland Sprague demonstrated in 1939 that also a square can be dissected in this way [Sprague 1939]. Four British mathematicians, Leonard Brooks, Cedric Smith, Arthur Stone and William Tutte, put forward, among other things, a new way to obtain possible dissections of

Arkadiusz Maciuk

Wrocław University of Economics e-mail: arkadiusz.maciuk@ue.wroc.pl ORCID: 0000-0001-5208-1074
Antoni Smoluk

Wrocław University of Economics

e-mail: math@ue.wroc.pl

ORCID: 0000-0002-9990-037X 
a rectangle into squares using the so-called Smith diagrams [Brooks et al. 1940]. As a result they proved that a firm relationship holds between dissecting a rectangle into squares and the current flowing in an electrical circuit with constant resistance. This relationship is a special example of the link between the theory of dissecting a rectangle into squares, and the theory of the current flows in isolated electrical networks with variable resistances. Since the 1960s, due to computer-aided applications and Smith diagrams, all possible dissections of rectangles into squares have been catalogued. Therefore it was demonstrated that the dissection of a square into fewer than 21 different squares is not possible, while A. J. W. Duijvestijn published the division of a square into 21 different squares [Duijvestijn 1978].

Perfect dissections of squares beautifully illustrate the strength of plane geometry. What is the law of nature, whose picture is given by a perfect squared square? The history of science teaches that formal laws sooner or later meet their reflection in nature. Perhaps Kirchhoff's principles represent this law of equilibrium. Fermat's problem is worth noting as an interesting aspect linked to the dissection of cubes. The two cubes cannot be dissected so as to be able to assemble one large cube using smaller ones. On a plane, a square can be divided into a finite number of different squares, but on higher order planes such dissection is not possible [Moron 1955; Anderson 2016].

\section{Squared rectangles}

A rectangle is an ordered pair $(x, y)$ of positive real numbers. Hereinafter, according to the convention introduced by Zbigniew Moron, we shall in fact consider rectangles with sides whose lengths are natural numbers. A division or a dissection of a rectangle $(k, n)$ into rectangles - where $k, n$ are nonzero natural numbers: $k$ denotes the length of the base, and $n$ denotes the height - is such a finite family of rectangles whose side lengths are also natural numbers, and which can produce the original rectangle $(k, n)$. The arrangements of rectangles with given side lengths can be a useful intellectual pleasure; they are a type of rectangular puzzles. Let $m_{k, n}$ denote the number of possible dissections of a rectangle $(k, n)$ into smaller rectangles with integer side lengths. An infinite matrix $M=\left(m_{k, n}\right)$ is mysterious in the sense that the recursive formulas to compute its terms are not available. It is only simple to compute the terms from its first column and the first row, as we have $c_{1, n}=c_{n, 1}=2^{n-1}$. Certainly, it is a symmetric matrix $c_{n, k}=c_{k, n}$. 
Segments will be considered equivalent to their lengths. The commensurability of segments $a$ and $b$ means that there are another segment $c$ and natural numbers $k$ and $n$ such that $a=k c, b=n c$. The commensurability of segments implies the linear dependence of vectors in an infinite space of real numbers $\mathbb{R}$ over a field of rational numbers $\mathbb{Q}$. An algebraic basis for this space, or a Hamel basis, consists of the number 1 and a certain infinite set of irrational numbers. The segments $a$ and $b$ in this space are commensurable if and only if a linear subspace spanned over the set $\{a, b\}$ is one-dimensional. The segments $a_{0}, \ldots, a_{k}$ are commensurable if and only if a linear space spanned over the set $\left\{a_{0}, \ldots, a_{k}\right\}$ is one-dimensional.

Theorem (Dehn). If a rectangle can be tiled using a finite set of squares, then the sides of this rectangle are commensurable, and the sides of squares are also commensurable.

Proof. Let us assume that a given set of squares can be used to tile a rectangle. We need to show that the sides of these squares are commensurable. Let the following $x_{0}, \ldots, x_{r}$ be the lengths of the unequal sides of these squares. We assume that there are $r$ unequal side lengths and that they are numbered in an increasing order, i.e. $x_{i}<x_{i+1}$. There are at least $r+1$ squares. We assume that a rectangle $(a, b)$ has been tiled by these squares. Given a real number $c \in[0, a]$, then a straight line $x=c$ does not include any side of squares, excluding the finite number equal $c$. The side lengths of squares crossed by the straight line add up in the equation $k_{0, c} x_{0}+\cdots+k_{r, c} x_{r}=b$, where $k_{i, c}$ denotes the number of squares with side $x_{i}$ crossed by the line $x=c$. There are infinitely many values c, but the number of independent equations is finite. We assume that there are $p$ equations. They are of the form: $n_{i, 0} x_{0}+\cdots+n_{i, r} x_{r}=b$, where $i \in\{1, \ldots, p\}$. Similarly, for $y \in[0, b]$ we get a system of $q$ independent equations of the form $m_{j, 0} x_{0}+\cdots+m_{j, r} x_{r}=a$, where $j \in\{1, \ldots, q\}$. These two systems of equations combined yield a determined system of equations. Remember that both side $a$ and side $b$ are sums of some sides of squares. Substituting $a$ and $b$ by these sums, we get a homogenous system of linear equations. Finally, we consider the quantity $x_{0}$ as given, and other quantities are unknowns. They are expressed in a rational manner by the length $x_{0}$, which was to be demonstrated.

Let us consider the three following dissections illustrated in Figure 1. The first dissection is described by linear relationships: $4 x_{0}=3 x_{1}=x_{2}=a$ and $4 x_{0}+x_{1}+x_{2}=b$, hence $x_{1}=\frac{4}{3} x_{0}, x_{2}=4 x_{0}$. In the second case, we have: 
$2 x_{0}+x_{2}=3 x_{1}=2 x_{2}=x_{3}=a$ and $2 x_{0}+x_{1}+x_{2}+x_{3}=x_{1}+2 x_{2}$ $+x_{3}=b$. These relationships, after simple rewriting, are reduced to the form of the system of three independent equations with three unknowns: $x_{1}, x_{2}, x_{3}\left\{\begin{aligned} 3 x_{1}-x_{3} & =0 \\ x_{2}-x_{3} & =-2 x_{0}, \\ x_{2} & =2 x_{0}\end{aligned}\right.$ which has a unique solution $x_{1}=\frac{4}{3} x_{0}$, $x_{2}=2 x_{0}, x_{3}=4 x_{0}$. The equations established by horizontal partitions of the third dissection are: $x_{2}+x_{4}+x_{5}=2 x_{0}+x_{2}+x_{3}+x_{4}=2 x_{0}+2 x_{1}+$ $x_{2}+x_{3}=2 x_{1}+x_{2}+2 x_{3}=2 x_{1}+x_{2}+2 x_{3}$; and by vertical partitions: $4 x_{2}=2 x_{1}+2 x_{4}=2 x_{3}+x_{5}=2 x_{0}+x_{3}+x_{5}$.
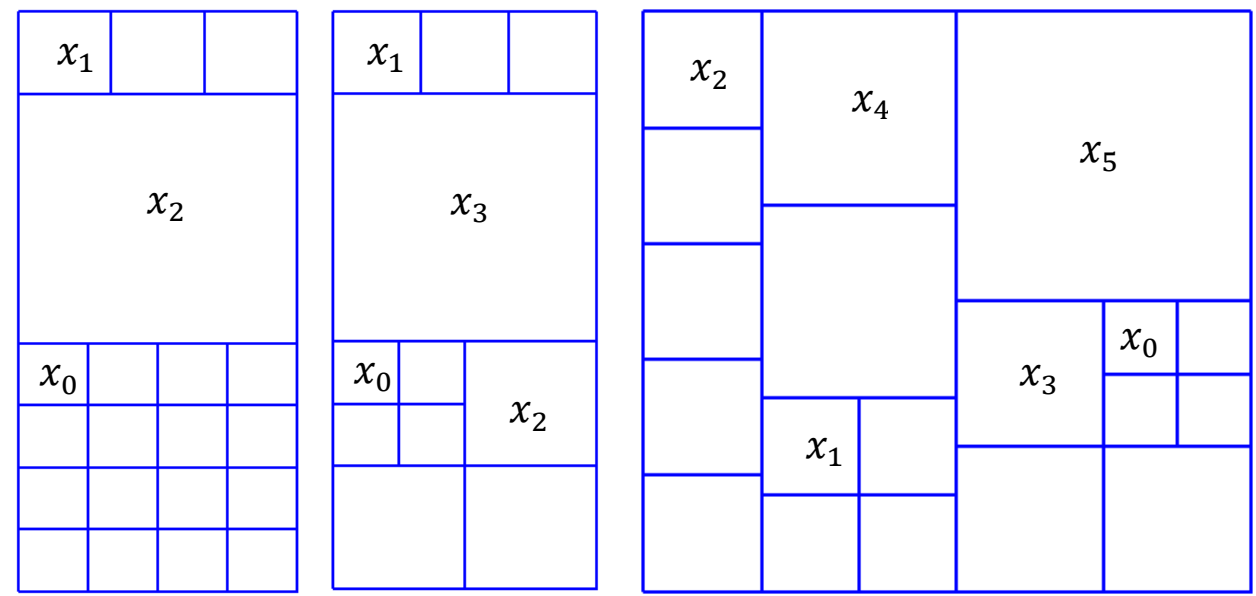

Fig 1. Illustration of the theorem on commensurability

Source: own elaboration.

The theorem implies that with a proper unit of length one can consider the side lengths of this rectangle as natural numbers. A dissection of a finite family of squares is called a division of some squares from this family into $4,9,16$, etc. smaller, equal squares. If the sides of a given set of squares are commensurable, then after any dissection of this family we get a larger family of squares, also with commensurable sides. A dissection preserves the invariant commensurability of the sides of squares from a given family: if the sides are commensurable, they are still commensurable after dissection; if they are not commensurable, they remain incommensurable after dissection.

Corollary. A finite family of squares has commensurable sides if and only if a rectangle can be tiled by this family up to dissection.

This corollary results from the theorem and remarks above. 
The construction presented in the proof above was applied by the first researchers of this issue and is still applied currently using integer programming algorithms [Kurz 2012]. Another, equivalent method proposed originally by Brooks et al. [1940] is implemented into designing electrical networks and sewerage systems, it can also be utilized in issues related to the optimal design of transmission networks and researching relationships in existing networks.

\section{Smith diagrams}

Kirchhoff's laws for electrical networks formulated in 1847 by the German physicist allow to determine the value and direction of currents flowing in an electrical circuit, as well as the potential difference (or electric tension) between the two selected nodes in the circuit. They can be expressed in the following simplest way. Kirchhoff's current law - the sum of all currents entering a node is equal to the sum of all currents leaving the node, and Kirchhoff's voltage law - the directed sum of the electrical potential differences around a loop must be zero. In 1940, four English mathematicians, Leonard Brooks, Cedric Smith, Arthur Stone and William Tutte, explained the correspondence between an electrical network with equal resistances and squared rectangles.

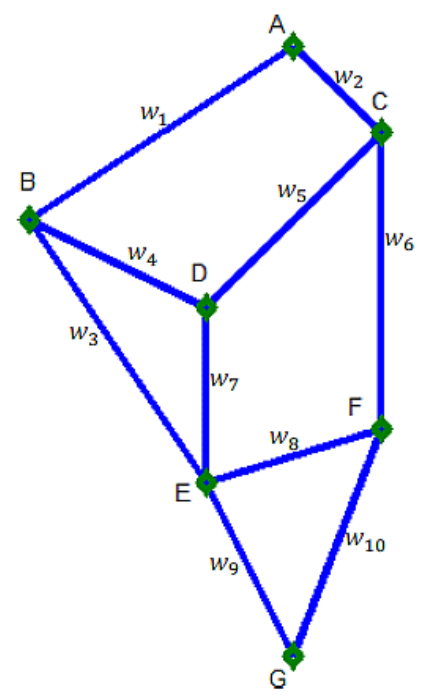

Fig. 2. An example of an electric circuit

Source: own elaboration. 
An electrical network can be presented by a graph with two particular vertices, called respectively a transmitter (source), and a receiver (ground), whose each $j^{\text {th }}$ linkage is characterized by a vector of parameters $w_{j}=\left(i_{j}, u_{j}, r_{j}\right)$, where $i_{j}$ is the coefficient of electric current, $u_{j}-$ of electric tension, and $r_{j}$ - of resistance. The direction of the flow of the electric current is assumed to be significant. When the direction complies with the orientation North-South, then the electric current is positive, otherwise it is negative. Let us consider the network presented in Figure 2. The connection AB has a positive orientation, while BA - negative. It follows from Kirchhoff's first law that each node, except for the transmitter and receiver, is associated with one equation expressing the relationship between the given currents. In the case of the network presented in Figure 2, these equations are of the form:

$$
\begin{aligned}
& B \quad i_{1}=i_{3}+i_{4} \\
& \text { C } \quad i_{2}=i_{5}+i_{6} \\
& \text { D } \quad i_{4}+i_{5}=i_{7} \\
& E i_{3}+i_{7}+i_{8}=i_{9} \text {, } \\
& F \quad i_{6}+i_{8}=i_{10} \text {. }
\end{aligned}
$$

Kirchhoff's second law describes the relationships between respective currents. Each cordless cycle determines one equation. Hence, we have

$$
\begin{aligned}
& A B D C A u_{1}+u_{4}=u_{2}+u_{5}, \\
& \text { BEDB } u_{3}=u_{4}+u_{7}, \\
& \text { CDEFC } u_{5}+u_{7}=u_{6}+u_{8}, \\
& \text { EGFE } u_{8}+u_{9}=u_{10} .
\end{aligned}
$$

The equations of the remaining cycles will be linearly dependent over the equations determined by the chordless cycles. For example, the equation of the ABEDCA cycle is the sum of equations of the ABDCA cycle and the BEDB cycle. We naturally assume that electric tension is in direct proportion with the current, i.e. $u_{j}=i_{j} r_{j}$. Hence, a system of equations is obtained:

$$
\begin{aligned}
i_{1}-i_{3}-i_{4} & =0 \\
i_{2}-i_{5}-i_{6} & =0 \\
i_{4}+i_{5}-i_{7} & =0 \\
i_{3}+i_{7}+i_{8}-i_{9} & =0 \\
i_{6}+i_{8}-i_{10} & =0 \\
i_{1} r_{1}-i_{2} r_{2}+i_{4} r_{4}-i_{5} r_{5} & =0 \\
i_{3} r_{3}-i_{4} r_{4}-i_{7} r_{7} & =0 \\
i_{5} r_{5}-i_{6} r_{6}+i_{7} r_{7}-i_{8} r_{8} & =0 \\
i_{8} r_{8}+i_{9} r_{9}-i_{10} r_{10} & =0 .
\end{aligned}
$$


Given the quantities of resistances $r_{1}, r_{2}, \ldots, r_{10}$, electric currents $i_{1}, i_{2}, \ldots, i_{9}$ can be expressed as a linear combination $i_{10}$.

The choice of a transmitter and a receiver unambiguously determines the directions and weights of the connections. Each Kirchhoff network consisting of $n$ links is defined by a system of $n-1$ independent linear equations: $n-1$ weights depend on the $n^{\text {th }}$ weight. Brooks et al. [1940] demonstrated that solving such a system of equations is equivalent to dissecting a rectangle into n squares, where respectively $u_{j}$ corresponds with the height, and $i_{j}$ with the width of the $i^{\text {th }}$ rectangle. If all the values of resistances equal 1 (i.e. when the current equals the tension of each link), we obtain a squared rectangle. In the example of the network shown in Figure 2, the currents equal respectively:

$$
\begin{gathered}
i_{1}=\frac{23}{22} i_{10}, i_{2}=\frac{12}{11} i_{10}, i_{3}=\frac{17}{22} i_{10}, i_{4}=\frac{3}{11} i_{10}, i_{5}=\frac{5}{22} i_{10}, \\
i_{6}=\frac{19}{22} i_{10}, i_{7}=\frac{1}{2} i_{10}, i_{8}=-\frac{3}{22} i_{10}, i_{9}=\frac{25}{22} i_{10} .
\end{gathered}
$$

Current $i_{8}$ is negative, thus meaning that the current flows from point $\mathrm{E}$ to point $\mathrm{F}$, contrarily to the figure shown. The squared rectangle corresponding to this scheme is presented in the following figure (next to $i_{10}=22$ ). This method is called the Smith diagrams method after its author [Anderson 2016]. Each diagram corresponds to another dissection, and each dissection to another diagram. As a result one can catalogue all possible rectangle squarings.

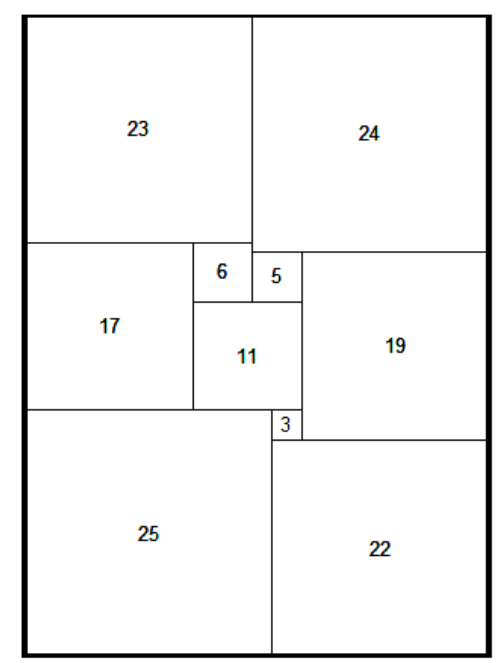

Fig 3. A squared rectangle corresponding to the electric circuit in Figure 2

Source: own elaboration. 
A typical economic issue related to this problem is transportation network planning so as to avoid blockages. A link in an electrical network becomes a route in a transportation network, electric tension - speed of traveling a route, and current - the load of commodities or passengers. The ratio of speed to load of commodities is route capacity. There is no congestion if the load of commodities entering a given node in a certain time unit equals the load of commodities leaving this node, and the speed of traveling two different routes - which make any chordless cycle is the same. This corresponds to researching the current in isolated electrical networks with equal resistances.

This method can be employed to investigate relationships that might be presented by graphs. Let us consider the problem of delivering goods from a producer to a receiver by means of various processing brokers, as illustrated by the graph in Figure 2. Vertex A represents a producer, $G$ - a receiver, vertices $B, C, D, E$, and F represent brokers. Goods are processed in two steps. Brokers B, C and D deal with the first step, brokers $\mathrm{E}$ and $\mathrm{F}$ - with the second step, whereas goods are distributed so as to avoid costs of deadlocks and storage. It is also assumed that the network of these connections is stable, that the amount of received goods depends on the processing capacity of the brokers, and the processing capacities match the loads received. A producer dispatches goods to two brokers; $\mathrm{B}$ and $\mathrm{C}$. Their processing capacities are limited, and it follows from the shape of the graph that they receive more goods than they can process, therefore they transfer a certain surplus to broker D. Preprocessed goods are conveyed to brokers $\mathrm{E}$ and $\mathrm{F}$, whereas $\mathrm{E}$ collects goods from $\mathrm{B}$ and $\mathrm{D}$, and $\mathrm{F}$ from $\mathrm{C}$ only. The graph also shows that there is a relationship between them, but it is not clear whether the surplus is conveyed by $\mathrm{F}$ to $\mathrm{E}$, or vice versa.

The computational results indicate that broker B receives 23/47 of goods produced by A, processes $17 / 23$ of this amount and transfers to broker E for more processing, while the unprocessed fraction (6/23) is sent to broker D. Broker C respectively takes delivery of $24 / 47$, processes $19 / 24$ of this amount and sends it to F, while the remainder is transferred to D. Broker D processes $11 / 47$ of the entire load and sends it to E. Broker E obtains 28/47, processes $25 / 28$ of this amount, and broker $F$ is given 22/47, including 3/47 of the surplus sent by broker $\mathrm{E}$.

\section{Simple perfect squared rectangles}

A square is clearly a special rectangle. Among dissections of a rectangle into rectangles one can distinguish dissections of a rectangle into squares. 
What condition must be satisfied by a rectangle $(k, n)$ for its dissections into squares to be possible? If we allow identical squares, then clearly any rectangle with commensurable sides can be tiled by squares. Among possible tilings there also exist such solutions that all squares have different dimensions. Such a tiling is called perfect squaring. Dividing a rectangle into unequal squares is a simpler task than dividing a square into unequal squares.

Dissecting both rectangles as well as squares is associated with the partitioning of the sides of a rectangle or a square. The question concerning the existence of the dissection of a rectangle into unequal squares is related to the nature of the partitions of its sides. The equations discussed above, concerning rectangle squaring, are in fact the partitions of the sides of this rectangle. For the sake of exemplification, we write these equations using the case of the perfect squared rectangle given by Moron [1925] (see Figure 4). Equations being horizontal partitions have the following form in dissection given by Moroń:

$$
\begin{aligned}
& x_{4}+x_{5}+x_{6}=x_{7}+x_{8} \\
& x_{0}+x_{3}+x_{5}+x_{6}=x_{7}+x_{8} \\
& x_{1}+x_{2}+x_{3}+x_{6}=x_{7}+x_{8} \\
& x_{2}+x_{3}+x_{8}=x_{7}+x_{8}
\end{aligned}
$$

whereas vertical partitions are equations of the form:

$$
\begin{aligned}
& x_{1}+x_{5}+x_{8}=x_{6}+x_{8} \\
& x_{2}+x_{5}+x_{7}=x_{6}+x_{8} \\
& x_{0}+x_{2}+x_{4}+x_{7}=x_{6}+x_{8} \\
& x_{3}+x_{4}+x_{7}=x_{6}+x_{8} .
\end{aligned}
$$




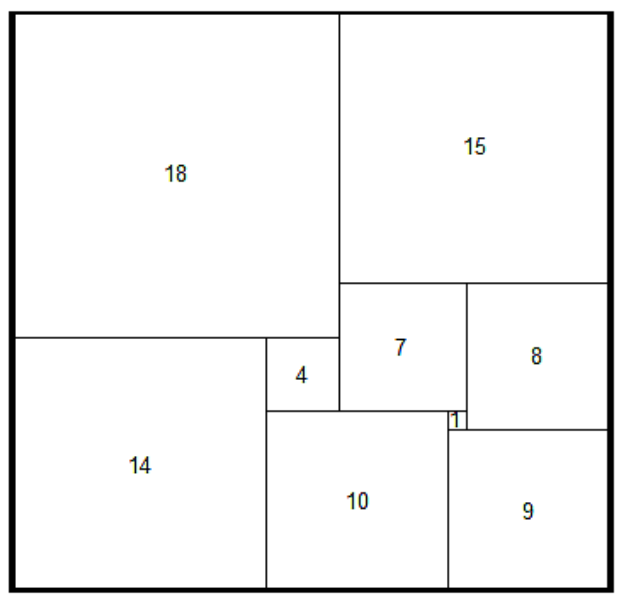

Fig. 4. Moroń perfect squared rectangle 33x32

Source: own elaboration.

After rewriting and using a matrix notation, we obtain a system:

$$
\left(\begin{array}{ccccccccc}
0 & 0 & 0 & 0 & 1 & 1 & 1 & -1 & -1 \\
1 & 0 & 0 & 1 & 0 & 1 & 1 & -1 & -1 \\
0 & 1 & 1 & 1 & 0 & 0 & 1 & -1 & -1 \\
0 & 0 & 1 & 1 & 0 & 0 & 0 & -1 & 0 \\
0 & 1 & 0 & 0 & 0 & 1 & -1 & 0 & 0 \\
0 & 0 & 1 & 0 & 0 & 1 & -1 & 1 & -1 \\
1 & 0 & 1 & 0 & 1 & 0 & -1 & 1 & -1 \\
0 & 0 & 0 & 1 & 1 & 0 & -1 & 1 & -1
\end{array}\right) .
$$

If we divide a rectangle into $r+1$ unequal squares, the partitions of sides must consist of different terms; none of the numbers from the set $\{1, \ldots, r+1\}$ can reappear in these partitions.

Remark. If a rectangle $(k, n)$ can be tiled by $r+1$ unequal squares, then $r+1 \leq \max \{k, n\}$.

There exist rectangles, including squares, that can be divided into unequal squares. Clearly the question is about the smallest rectangle and the smallest square with this property. If a square with side $n$ can be divided into $r+1$ unequal squares, then the number $n$ belongs to the set $\mathbb{N}_{S}$, and the number $r+1$ to the set $\mathbb{N}_{D}$; there are no other numbers in these sets. Obviously these sets are not empty, and from the previous findings we conclude that 
$\min \mathbb{N}_{D}=21 \leq \min \mathbb{N}_{S}=110$ [Anderson 2016]. A square divided into the smallest number of unequal squares, i.e. 21 , has the side equal to 112 , whereas a rectangle with the shortest side that can be divided into 22 unequal squares has the side equal to 110 [Anderson 2016; Duijvestijn 1978]. Thus, a square with the shortest side cannot be tiled by the smallest number of unequal squares.

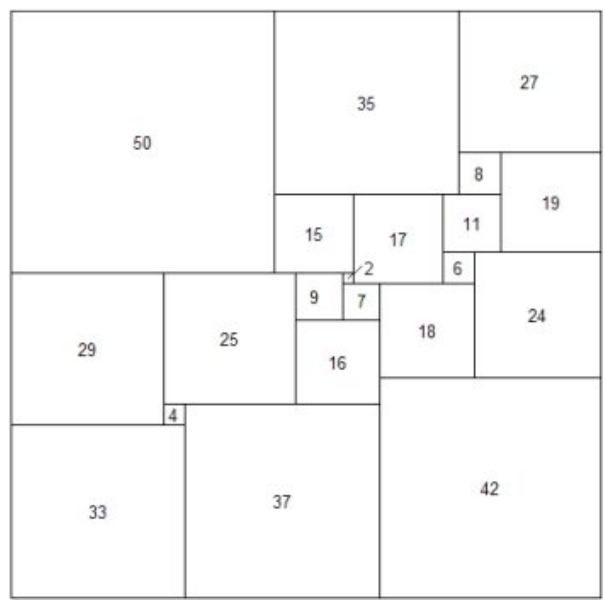

Fig. 5. Duijvestijn squared square $112 x 112$ of order 21

Source: [Anderson 2016].

Let us assume that a certain square with a side $n$ has a tiling by $r+1$ unequal squares with sides $x_{0}, \ldots, x_{r}$; the sides are numbered increasingly. Furthermore, let natural numbers $n_{E}, n_{W}, n_{N}, n_{S}$ denote the numbers of partitions respectively of the eastern, western, northern and southern side; they are the numbers of squares adjacent to the respective sides. Certainly, the two dissections which are either symmetric vertically or horizontally, or obtained by rotating any one of them through an angle of $\frac{\pi}{2}$, are considered identical. These four numbers do not determine a dissection of squares into unequal squares [Anderson 2016]. For example, a square with partitions of sides $n_{E}=3, n_{W}=3, n_{N}=3, n_{S}=4$ can be divided into 21 sections, there is one division among 8 possible ones into 22 sections, three divisions out of the possible 12 into 23 sections, and six of the possible 26 into 24 unequal sections.

A square can be divided into a finite number of unequal squares, but a cube cannot - dividing a cube into unequal cubes is not possible. Dimensions greater than 2 have a more intricate nature. The properties true in the 
case of plane objects need not to be valid in higher dimensions. This is also the case regarding the perfect squaring of squares that has no equivalent in the tiling of cubes. Scientific truths should have their counterparts in the real world. What corresponds in nature to the perfect squaring of rectangles and squares? Undoubtedly such a correspondence exists. There have been some attempts to explain it by means of analogies to electricity and to mechanical transfers of objects, nevertheless a complete correspondence is still missing. The mystery of perfect dissections is still out there waiting to be explained.

\section{Bibliography}

Anderson S. (2016). Squaring.Net. http://www.squaring.net/.

Brooks R.L., Smith C.A., Stone A.H., Tutte W.T. (1940). The dissection of rectangles into squares. Duke Mathematical Journal. No. 7. Pp. 213-340.

Dehn M. (1903). Über Zerlegung von Rechtecken in Rechtecke. Math. Annalen. No. 57. Pp. 314-332.

Duijvestijn A.J.W. (1978). Simple perfect square of lowest order. Journal of Combinational Theory. Ser. B. No. 25. Pp. 240-243.

Kurz S. (2012). Squaring the square with integer linear programming. Journal of Information Processing. No. 20(3). Pp. 680-685.

Moroń Z. (1925). O rozkladach prostokątów na kwadraty. Przegląd Matematyczno-Fizyczny. No. 3. Pp. 152-153.

Moroń Z. (1955). O rozkladach prostokątów na nierówne kwadraty. Wiadomości Matematyczne. No. 1(1). Pp. 75-94.

Sprague R. (1939). Beispiel einer Zerlegung des Quadrats in lauter verschiedene Quadrate, Mathematische Zeitschrift. No. 45. Pp. 607-608. 